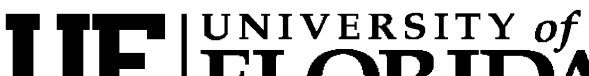 FLORIDA \\ IFAS Extension
}

ENH1118

\section{Selected Miticides for Use on Ornamental Plants ${ }^{1}$}

\section{Robert H. Stamps and Lance S. Osborne ${ }^{2}$}

Mites are among the most difficult arthropod pests to control on ornamental plants. Adult mites have eight legs and piercing/sucking mouthparts that are used to suck fluids from the cells of host plants (Denmark, 1969). The first immature stage of a mite, referred to as the larva, has only six legs, like insects. The exceptions are the Eriophyid mites, which have four legs in all stages. Mites are not insects, but are more closely related to spiders and ticks. Thousands of species of mites feed on plants.

Spider mites, members of the Tetranychidae family, are perhaps the most important mite pests of ornamental plants. The name, spider mites, is due to the many members of this family that produce silk webbing. Spider mites are medium-sized mites that feed on a wide variety of host plants from many different plant families. Some spider mites are bamboo, Lewis, southern red, spruce, tumid and twospotted mites.

Members of the false spider mites family, Tenuipalpidae, do not produce silk webbing, but a number of these species feed on ornamental plants. False spider mites are generally smaller than spider mites. Examples of false spider mites are flat and red palm mites.
Some Tarsonemid mites (family Tarsonemidae) are smaller than even false spider mites. This family includes broad and cyclamen mites.

Eriophyid mites (Eriophyidae family) are too small to be seen with the naked eye and include bud, gall, purple tea and rust mites, among others. As their names suggest, these mites can cause galls, rusts and other abnormal plant growth.

There are other families of mites that have crop-damaging members, but the mites named above are the main mite pests of ornamental plants.

Mites of a given species can develop very rapidly when temperatures, relative humidities, host plants and other factors are optimal. In fact, for many, the time to develop from an egg to an adult can be less than a week. Generally, development occurs more rapidly at higher temperatures, up to a point. Due to mites rapid development, scouting should be performed frequently (at least once per week), and miticide applications may need to be made on weekly or more-frequent intervals (be sure to check the miticide labels for instructions and restrictions associated with spray intervals) during the summer.

1. This document is ENH1118, one of a series of the Environmental Horticulture Department, Florida Cooperative Extension Service, Institute of Food and Agricultural Sciences, University of Florida. Original publication date, April 2009. Visit the EDIS Web site at http://edis.ifas.ufl.edu.

2. Robert H. Stamps, professor, Environmental Horticulture Department, and Lance S. Osborne, professor, Entomology and Nematology Department, and associate director, Mid-Florida Research and Education Center--Apopka, FL, Institute of Food and Agricultural Sciences, University of Florida. 


\section{Detection}

Frequent, careful inspection of plants is necessary to detect mite infestations before they reach epidemic levels and cause severe plant damage. By the time plant symptoms become very obvious to the unaided eye, control of the mites will be difficult and potentially expensive. Early detection can limit damage and facilitate economical control of mites. Careful inspection is necessary because damage due to mites can, on some plants, resemble that from other causes. For example, symptoms can look similar to insect feeding, nutritional deficiencies, physiological stress, herbicide damage, etc.

Since many mites feed on the undersides of leaves, these are important sites to check. Spider mites can usually be detected on older leaves, whereas Tarsonemids are often found on young leaves. False spider mites often feed near the midrib or veins. Silvery speckling/stippling of the upper leaf surface is a characteristic symptom of leaf feeding. Fine thread-like webbing may also be present (spider mites). Mites may also feed on petioles, stems, buds and other plant parts. Mite feeding can cause a multitude of symptoms, such as leaf cupping, discoloration, distortion, spotting, speckling and stunting, stem russeting and discoloration, as well as distorted and discolored flowers.

Because mites are so small, the use of a magnifying hand lens $(10 \mathrm{x})$ will make it easier to observe them. Another technique frequently used to detect mites, especially on crops with fine foliage like ornamental asparagus, is to slap stems firmly on a light-colored surface, such as a white painter`s palette or a sheet of white paper on a clipboard. If mites are present, they will be easily seen as small, moving spots.

\section{Control}

Both biological control agents and miticides are available for controlling mites. For example, the predatory mites, Neoseiulus californicus and Phytoseiulus persimilis, can effectively control twospotted spider mites. At least 15 other predators are commercially available for control of this and other pests (Osborne and Peña, 1997). P. persimilis has been used successfully to control twospotted spider mites on many plants growing in protected culture (greenhouses and shadehouses). $N$. californicus is just as effective, and it tolerates pesticide residues better than $P$. persimilis and also feeds on broad mites [Polyphagotarsonemus latus (Banks)]. For more information on biological control of mites, refer to the following Web site: http://mrec.ifas.ufl.edu/lso/SpMite/b853a1.htm.

A listing of commercial suppliers of biological mite control agents is available at the following Web site:

http://www.cdpr.ca.gov/docs/pestmgt/ipminov/ ben_supp/contents.htm.

Besides frequently scouting crops to detect mite infestations early, growers should avoid using pesticides that are harmful to eggs, immatures and adults of predatory mites. Companies that supply mite predators furnish customers with lists delineating the effects of pesticides on the predators. (See "side effects" at the following Web site: http://www.biobest.be and http://www.koppert.nl/e005.shtml)].

For chemical mite control, the use of broad-spectrum insecticides/miticides (especially products with long-lived residual activity, such as the pyrethroids listed in Table 1) is generally not recommended. These insecticides/miticides may directly or indirectly harm beneficial insects and/or mites, and a rapid increase in existing plant-feeding mite populations may result. Use of the insecticide carbaryl is also known to occasionally increase twospotted spider mite infestations on susceptible plants. Therefore, selective chemicals that specifically target plant-feeding mites should be used.

Predatory mites must be released as soon as pest mites are detected, and the number of predatory mites released must be sufficient to ensure control of the pest mites (Osborne et al., 1985). As mentioned previously, check with biological control suppliers for guidance and to find out which predatory-mite strains are resistant or susceptible to specific pesticides. This information can help in making decisions regarding pesticide use. For more information on biological control, see Osborne and Peña, 1997. 
Although mites can become resistant to miticides; several techniques can reduce the likelihood of this happening. First, minimize miticide usage by incorporating biological and cultural methods into your pest management program. Practice good sanitation methods and use mite resistant crops and varieties. Scout frequently (at least once a week) and only apply miticides when necessary. Design growing areas so all areas can be easily and effectively treated.

Do not use miticides with the same mode of action in succession. Rather, practice long-term rotations, using as many products with different modes of action as possible. For example, carbamates and organophosphates both work by inhibiting the enzyme acetylcholinesterase, which is important in the proper functioning of the nervous system. Most of the other classes of miticides have different modes of action. Table 1 lists miticides commonly used on ornamental crops and the mode of action groups for these miticides. Active ingredients, trade names, formulations and labeling (application locations, restricted use designations, restricted entry intervals [REIs] and manufacturer/distributors' names are also given. Users of any pesticide should make sure they have the current label since labels may change over time. In addition, users should read labels in their entirety and comply with the label`s directions and restrictions. Table 2 lists the addresses of the manufacturers/distributors of the miticides listed in Table 1. Another useful strategy for controlling mites is to include products with a non-specific mode of action, where possible, into a rotation. This group includes insecticidal soaps and horticultural oils.

Miticides should only be used as labeled ("the label is the law"). Fortunately, some miticides have general labeling that allows them to be used, at the grower`s risk, on a broad range of crops not specifically listed on the label. Prudent growers should test multiple applications of any miticide if it is likely that it will be used more than once. In addition, to enhance miticide efficacy, surfactants and adjuvants maybe included if there are no label restrictions.

Allow sufficient time after treating the test plants for phytotoxicity symptoms to develop. If a new miticide will be used in tank mixes or in close sequences with other pesticides, evaluate the potential for plant injury (phytotoxicity) on a small number (about 10) of plants of each species and cultivar before treating all the plants of each crop. These tolerance tests should take into consideration the potential interactions with other pesticides used in the pest control program.

It is essential to read pesticide labels since all restrictions related to where each product can be used (greenhouse, shadehouse, interiorscape, outdoor field nursery, outdoor nursery, landscape, residential landscape), how it can be applied (chemigation, spray, aerial, etc.), on what growing media it can be used, etc., must be observed. State and local labeling may differ from and be more restrictive than federal labeling. Pesticide applicators should always review product labels before using any pesticide and have all pertinent labels (including supplemental labels) in their possession prior to use of any pesticide.

\section{Selected References}

Denmark, H. A. 1969. Two-spotted spider mite on chrysanthemum. Fla. Dept. of Agr. and Consumer Serv., Div. of Plant Industry, Ento. Circ. No. 89.

Osborne, L. S., L. E. Ehler and J. R. Nechols. 1985. Biological control of the twospotted spider mite in greenhouses. Univ. of Fla., Inst. of Food and Agr. Sci., Agr. Expt. Sta. Bul. 853 (technical) http://mrec.ifas.ufl.edu/lso/SpMite/b853a1.htm.

Osborne, L. S. and J. Peña. 1997. More than you want to know about mites and their biological control on ornamentals. Proc. of the 13th Society of American Florists ${ }^{`}$ Conference on Insect and Disease Management on Ornamentals. pp. 53-85.

Sabelis, M. W. 1981. Biological control of twospotted spider mites using phytoseiid predators. I. Agric. Res. Report 910, Pudoc, Wageningen, the Netherlands.

Zhang, Z. 2003. Mites of Greenhouses: Identification, Biology and Control. CABI Publishing, Wallingford, Oxfordshire, UK. 
Table 1. Selected products for use in controlling mites on ornamental plants.

\begin{tabular}{|c|c|c|c|c|c|c|c|c|c|c|}
\hline $\begin{array}{l}\text { Mode } \\
\text { of } \\
\text { action } \\
\text { group }^{z}\end{array}$ & $\begin{array}{l}\text { Active } \\
\text { ingredient }\end{array}$ & Trade name(s) & Formulation(s) & $\begin{array}{c}\text { Mites } \\
\text { controlled }^{y}\end{array}$ & $\begin{array}{c}\text { Mite } \\
\text { stages } \\
\text { controlled }^{\mathrm{x}}\end{array}$ & Location $^{\text {w }}$ & $\begin{array}{c}\text { Broad } \\
\text { crop } \\
\text { labeling }\end{array}$ & $\begin{array}{c}\text { REI }^{v} \\
\text { (hours) }\end{array}$ & $\begin{array}{c}\text { Manufacturerl } \\
\text { Distributor }^{\mathrm{u}}\end{array}$ & Comments \\
\hline $1 \mathrm{~A}$ & carbaryl & $\begin{array}{l}\text { Carbaryl, } \\
\text { Sevin }^{\circledR}\end{array}$ & $\begin{array}{l}80 \mathrm{~S}, 4 \mathrm{SL}(43 \% \\
\left.\mathrm{ai}^{\mathrm{S}}\right), 4 \mathrm{~S} \\
(44.1 \%)\end{array}$ & Eriophyid & & $\mathrm{O}$ & Yes & 12 & $\begin{array}{l}\text { Drexel, Bayer, } \\
\text { Prokoz, others }\end{array}$ & $\begin{array}{l}\text { Thorough coverage of } \\
\text { upper- and lower-leaf } \\
\text { surfaces is important. Use } \\
\text { may increase twospotted } \\
\text { spider mite populations. }\end{array}$ \\
\hline \multirow[t]{2}{*}{ 1B } & diazinon & $\begin{array}{l}\underbrace{\mathrm{t}}{ }^{\mathrm{Diazinon}}{ }^{\circledR} \\
\text { AG500 }\end{array}$ & 4 EC (48\% ai) & $\begin{array}{l}\text { Certain } \\
\text { spider mites, } \\
\text { cyclamen, } \\
\text { mites, } \\
\text { carnation } \\
\text { bud and } \\
\text { shoot mites }\end{array}$ & & On & No & 12 & Helena & $\begin{array}{l}\text { Only for certain labeled } \\
\text { crops. }\end{array}$ \\
\hline & disulfoton & $\underbrace{}_{x}$ Di-Syston ${ }^{\circledR}$ & $15 \mathrm{G}$ & & & $\mathrm{O}$ & No & 48 & Bayer & $\begin{array}{l}\text { Labeled only for use on firs } \\
\text { (Christmas trees). }\end{array}$ \\
\hline \multirow[t]{2}{*}{$2 A$} & endosulfan & 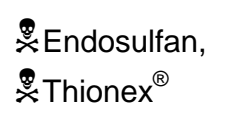 & $\begin{array}{l}3 \text { EC (33.7- } \\
34.0 \% \text { ai) }\end{array}$ & $\begin{array}{l}\text { Taxus bud } \\
\text { mite }\end{array}$ & $\mathrm{I}, \mathrm{A}$ & On & No & 48,24 & $\begin{array}{l}\text { Drexel, } \\
\text { Makhteshim- } \\
\text { Agan }\end{array}$ & Shrubs and trees. \\
\hline & & $\underset{x}{*}$ Thionex $^{\circledR}$ & $50 \mathrm{~W}$ & $\begin{array}{l}\text { Taxus bud } \\
\text { mite, } \\
\text { cyclamen }\end{array}$ & & On & No & 24 & $\begin{array}{l}\text { Makhteshim- } \\
\text { Agan }\end{array}$ & \\
\hline $3^{r}$ & bifenthrin & Attain ${ }^{(B)}$ TR & aerosol (4\% ai) & Spider & & G & Yes & 12 & $\begin{array}{l}\text { Whitmire Micro- } \\
\text { Gen }\end{array}$ & $\begin{array}{l}\text { Total release aerosol. } \\
\text { Significant resistance has } \\
\text { been detected in some } \\
\text { populations of spider mites. }\end{array}$ \\
\hline
\end{tabular}


Table 1. Selected products for use in controlling mites on ornamental plants. (continued)

\begin{tabular}{|c|c|c|c|c|c|c|c|c|c|c|}
\hline $\begin{array}{l}\text { Mode } \\
\text { of } \\
\text { action } \\
\text { group }^{2}\end{array}$ & $\begin{array}{l}\text { Active } \\
\text { ingredient }\end{array}$ & Trade name(s) & Formulation(s) & $\begin{array}{c}\text { Mites } \\
\text { controlled }^{\mathrm{y}}\end{array}$ & $\begin{array}{c}\text { Mite } \\
\text { stages } \\
\text { controlled }^{\mathrm{x}} \\
\end{array}$ & Location $^{\mathrm{w}}$ & $\begin{array}{c}\text { Broad } \\
\text { crop } \\
\text { labeling }\end{array}$ & $\begin{array}{c}\text { REI }^{v} \\
\text { (hours) }\end{array}$ & $\begin{array}{c}\text { Manufacturerl } \\
\text { Distributor }^{\mathrm{u}}\end{array}$ & Comments \\
\hline & & $\begin{array}{l}\underset{x}{*} \text { Attain }^{\circledR} \\
\text { Bifenthrin, } \\
\text { Broadcide }^{\mathrm{TM}} \text {, } \\
\text { Talstar }^{\circledR}, \text { UP- } \\
\text { Star, etc. }\end{array}$ & $\begin{array}{l}0.67 \mathrm{~F}[\mathrm{SC}] \\
(7.9 \% \mathrm{ai})\end{array}$ & $\begin{array}{l}\text { Spider and } \\
\text { broad }\end{array}$ & & G, On, S & Yes & 12 & $\begin{array}{l}\text { Whitmire Micro- } \\
\text { Gen, Quali-Pro, } \\
\text { Regal, FMC, } \\
\text { United } \\
\text { Phosphorus }\end{array}$ & $\begin{array}{l}\text { Significant resistance has } \\
\text { been detected in some } \\
\text { populations of mites. }\end{array}$ \\
\hline & cyhalothrin & $\underset{x}{*}$ Scimitar $^{\circledR}$ & $\begin{array}{l}0.88 \mathrm{GC}(9.7 \% \\
\text { ai) }\end{array}$ & Spider & & $\mathrm{G}, \mathrm{I}, \mathrm{O}, \mathrm{S}$ & yes & 24 & Syngenta & $\begin{array}{l}\text { Significant resistance has } \\
\text { been detected in some } \\
\text { populations of mites. }\end{array}$ \\
\hline & fenpropathrin & $\stackrel{2}{X}$ Tame $^{\circledR}$ & $\begin{array}{l}2.4 \text { EC }(30.9 \% \\
\text { ai) }\end{array}$ & Spider & & $\mathrm{G}, \mathrm{I}, \mathrm{O}, \mathrm{S}$ & Yes & 24 & Valent & $\begin{array}{l}\text { Significant resistance has } \\
\text { been detected in some } \\
\text { populations of mites. }\end{array}$ \\
\hline \multirow[t]{2}{*}{6} & abamectin & $\begin{array}{l}\text { Abamectin, } \\
\text { Avid }^{\circledR}\end{array}$ & $\begin{array}{l}0.15 \text { EC }(1.9- \\
2 \% \text { ai) }\end{array}$ & $\begin{array}{l}\text { Eriophyid, } \\
\text { Spider, } \\
\text { Tarsonemid }\end{array}$ & $\mathrm{I}, \mathrm{A}$ & $\mathrm{G}, \mathrm{O}, \mathrm{S}$ & Yes & 12 & $\begin{array}{l}\text { Quali-pro, } \\
\text { Syngenta }\end{array}$ & $\begin{array}{l}\text { Translaminar, also } \\
\text { suppresses aphids, thrips } \\
\text { and whiteflies. Do not use } \\
\text { on Shasta daisies or ferns. } \\
\text { Resistance has been } \\
\text { detected in some } \\
\text { populations of twospotted } \\
\text { spider mites }\end{array}$ \\
\hline & milbemectin & $\underset{x}{x}$ Ultiflora $^{\mathrm{TM}}$ & $\begin{array}{l}0.0775 \text { EC (1\% } \\
\text { ai) }\end{array}$ & $\begin{array}{l}\text { Eriophyid, } \\
\text { Spider, } \\
\text { Tarsonemid, } \\
\text { Tenuipalpid }\end{array}$ & $E, I, A$ & Of & Yes & 12 & Gowan & $\begin{array}{l}\text { Apply no more than } 128 \mathrm{fl} \\
\text { oz per acre per year. }\end{array}$ \\
\hline $10 A^{q}$ & clofentezine & Ovation $^{\mathrm{TM}}$ & 1 SC (42\% ai) & Spider & $E, I$ & G, On, S & Yes & 12 & Scotts & $\begin{array}{l}\text { Only one application per } \\
\text { crop cycle. Good residual } \\
\text { control. Unstable in alkaline } \\
\text { solutions. Useful in IPM } \\
\text { programs. Bright magenta } \\
\text { in color, residue may be } \\
\text { noticeable. }\end{array}$ \\
\hline
\end{tabular}


Table 1. Selected products for use in controlling mites on ornamental plants. (continued)

\begin{tabular}{|c|c|c|c|c|c|c|c|c|c|c|}
\hline $\begin{array}{c}\text { Mode } \\
\text { of } \\
\text { action } \\
\text { group }^{z}\end{array}$ & $\begin{array}{l}\text { Active } \\
\text { ingredient }\end{array}$ & Trade name(s) & Formulation(s) & $\begin{array}{c}\text { Mites } \\
\text { controlled }^{\mathrm{y}}\end{array}$ & $\begin{array}{c}\text { Mite } \\
\text { stages } \\
\text { controlled }^{\mathrm{x}} \\
\end{array}$ & Location $^{\mathrm{w}}$ & $\begin{array}{c}\text { Broad } \\
\text { crop } \\
\text { labeling }\end{array}$ & $\begin{array}{c}\text { REI }^{v} \\
\text { (hours) }\end{array}$ & $\begin{array}{c}\text { Manufacturerl } \\
\text { Distributor }\end{array}$ & Comments \\
\hline & hexythiazox & Hexygon $^{\mathrm{TM}}$ & 50 WP & Spider & $\mathrm{E}, \mathrm{I}$ & $\begin{array}{c}\text { G, I, O, S } \\
\text { Can also } \\
\text { be used in } \\
\text { land- } \\
\text { scapes }\end{array}$ & Yes & 12 & Gowan & Provides residual control. \\
\hline $10 B^{r}$ & etoxazole & TetraSan & 5 WDG & $\begin{array}{l}\text { Spider, } \\
\text { Tenuipalpid }\end{array}$ & $E, I$ & $\begin{array}{c}\mathrm{G}, \mathrm{I}, \mathrm{Lr}, \mathrm{O} \\
\mathrm{S}\end{array}$ & Yes & 12 & Valent USA & $\begin{array}{l}\text { Translaminar activity, } \\
\text { useful in IPM programs. } \\
\text { Best used in early stages of } \\
\text { infestations. Use no more } \\
\text { than twice per cropping } \\
\text { cycle. Sterilizes adult mites. }\end{array}$ \\
\hline $12 B^{r}$ & $\begin{array}{l}\text { fenbutatin- } \\
\text { oxide (also } \\
\text { known as } \\
\text { hexakis) }\end{array}$ & $\begin{array}{l}\text { ProMITE } \\
\text { (formerly } \\
\text { Vendex) }\end{array}$ & 50 WP & Spider & I, A & $\mathrm{G}, \mathrm{O}$ & Yes & 48 & SePRO & $\begin{array}{l}\text { For best results, apply } \\
\text { when mite populations are } \\
\text { just beginning to build. } \\
\text { Thorough and complete } \\
\text { coverage is necessary for } \\
\text { optimum control. Performs } \\
\text { best when daily } \\
\text { temperature at application } \\
\text { averages above } 70^{\circ} \mathrm{F} \text {. May } \\
\text { be applied when } \\
\text { honeybees and beneficial } \\
\text { mites are present. }\end{array}$ \\
\hline 13 & chlorfenapyr & Pylon $^{(B)}$ & $2 \mathrm{SC}$ & $\begin{array}{l}\text { Eriophyid, } \\
\text { Spider, } \\
\text { Tarsonemid }\end{array}$ & I & G & No & 12 & $\mathrm{OHP}$ & $\begin{array}{l}\text { Translaminar activity. } \\
\text { Avoid applying to blooming } \\
\text { flowers. No more than three } \\
\text { applications (not more than } \\
\text { the } 41 \mathrm{fl} \text { oz of product per } \\
100 \text { gal per crop per } \\
\text { season or } 0.64 \text { lb ai per } \\
100 \text { gal per crop per } \\
\text { season) should be applied } \\
\text { during a crop growing cycle } \\
\text { (start to finish for one } \\
\text { ornamental crop). Note } \\
\text { sensitive plants on label. }\end{array}$ \\
\hline
\end{tabular}


Table 1. Selected products for use in controlling mites on ornamental plants. (continued)

\begin{tabular}{|c|c|c|c|c|c|c|c|c|c|c|}
\hline $\begin{array}{l}\text { Mode } \\
\text { of } \\
\text { action } \\
\text { group }^{z}\end{array}$ & $\begin{array}{l}\text { Active } \\
\text { ingredient }\end{array}$ & Trade name(s) & Formulation(s) & $\begin{array}{c}\text { Mites } \\
\text { controlled }^{y}\end{array}$ & $\begin{array}{c}\text { Mite } \\
\text { stages } \\
\text { controlled }^{\mathrm{x}} \\
\end{array}$ & Location $^{\text {w }}$ & $\begin{array}{l}\text { Broad } \\
\text { crop } \\
\text { labeling }\end{array}$ & $\begin{array}{c}\text { REI }^{v} \\
\text { (hours) }\end{array}$ & $\begin{array}{c}\text { Manufacturerl } \\
\text { Distributor }\end{array}$ & Comments \\
\hline $20 B$ & acequinocyl & Shuttle ${ }^{\mathrm{TM}}$ & $15 \mathrm{SC}$ & $\begin{array}{l}\text { Spider, } \\
\text { Tenuipalpid }\end{array}$ & E (some), I & G, On, S & Yes & 12 & $\begin{array}{l}\text { Arysta } \\
\text { LifeScience }\end{array}$ & $\begin{array}{l}\text { Compatible with IPM } \\
\text { programs. Do not apply } \\
\text { successive applications, } \\
\text { rotate with treatments } \\
\text { having different modes of } \\
\text { action. Not for use on mini- } \\
\text { roses. }\end{array}$ \\
\hline \multirow[t]{2}{*}{$21 \mathrm{~A}$} & fenpyroximate & Akari $^{(B)}$ & $5 \mathrm{SC}$ & $\begin{array}{l}\text { Eriophyid, } \\
\text { Spider } \\
\text { Tarsonemid }\end{array}$ & I & G, I, On & Yes & 12 & SePRO & $\begin{array}{l}\text { Do not apply more than } 48 \\
\text { fl oz per crop cycle or } \\
\text { growing season. }\end{array}$ \\
\hline & pyridaben & Sanmite $^{(B)}$ & 75 WP & $\begin{array}{l}\text { Spider, } \\
\text { Tarsonemid }\end{array}$ & I, A (some) & $\mathrm{G}, \mathrm{O}, \mathrm{S}$ & Yes & 12 & Scotts & $\begin{array}{l}\text { Do not exceed } 21.34 \text { oz per } \\
\text { acre per year. }\end{array}$ \\
\hline 23 & spiromesifen & $\begin{array}{l}\text { Judo }^{\mathrm{TM}} \\
\text { Forbid }^{\mathrm{TM}}\end{array}$ & $\begin{array}{l}4 \mathrm{~F}[\mathrm{SC}](45.2 \% \\
\text { ai) } \\
4 \mathrm{~F}[\mathrm{SC}](45.2 \% \\
\text { ai) }\end{array}$ & $\begin{array}{l}\text { Eriophyid, } \\
\text { Spider, } \\
\text { Tarsonemid, } \\
\text { Tenuipalpid }\end{array}$ & $\begin{array}{l}\mathrm{E}, \mathrm{I}, \mathrm{A} \\
\text { (some) }\end{array}$ & $\begin{array}{c}\text { G, On, S } \\
\text { Ol }\end{array}$ & Yes & $\mathrm{na}^{\mathrm{p}}$ & $\begin{array}{l}\text { OHP } \\
\text { Bayer }\end{array}$ & $\begin{array}{l}\text { Translaminar activity. Do } \\
\text { not apply more than three } \\
\text { times per season and do } \\
\text { not make successive } \\
\text { applications. Check Judo } \\
\text { Technical Bulletin at } \\
\text { www.OHP.com for list of } \\
\text { sensitive plants. }\end{array}$ \\
\hline$u^{r}$ & bifenazate & Floramite $^{(B)}$ & 2 SC (22.6\%) & $\begin{array}{l}\text { Spider, } \\
\text { Tenuipalpid }\end{array}$ & $\begin{array}{c}\mathrm{E} \text { (some) }, \mathrm{I}, \\
\mathrm{A}\end{array}$ & $\mathrm{G}, \mathrm{I}, \mathrm{O}, \mathrm{S}$ & Yes & 12 & $\mathrm{OHP}$ & $\begin{array}{l}\text { Compatible with IPM and } \\
\text { resistance management } \\
\text { programs. Do not apply } \\
\text { more than } 32 \text { fluid oz per } \\
\text { acre per year. Not effective } \\
\text { against eriophyid or } \\
\text { tarsonemid mites. Adjust } \\
\text { spray water pH to below } 7 \text {. }\end{array}$ \\
\hline \multirow[t]{2}{*}{$\overline{\text { oils }}^{r}$} & $\begin{array}{l}\text { oil; cottonseed, } \\
\text { clove, garlic }\end{array}$ & GC-Mite ${ }^{\mathrm{TM}}$ & 70 LC & Spider & $E, I, A$ & & Yes & & JH Biotech & $\begin{array}{l}\text { Good coverage important. } \\
\text { OMRI listed. }\end{array}$ \\
\hline & oil, neem & Triact $^{(B)}$ & $70 \mathrm{EC}$ & Spider & $E, I, A$ & $\mathrm{G}, \mathrm{O}$ & Yes & 4 & $\mathrm{OHP}$ & \\
\hline
\end{tabular}


Table 1. Selected products for use in controlling mites on ornamental plants. (continued)

\begin{tabular}{|c|c|c|c|c|c|c|c|c|c|c|}
\hline $\begin{array}{l}\text { Mode } \\
\text { of } \\
\text { action } \\
\text { group }^{z}\end{array}$ & $\begin{array}{l}\text { Active } \\
\text { ingredient }\end{array}$ & Trade name(s) & Formulation(s) & $\begin{array}{c}\text { Mites } \\
\text { controlled }^{y}\end{array}$ & $\begin{array}{c}\text { Mite } \\
\text { stages } \\
\text { controlled }^{\mathrm{x}}\end{array}$ & Location $^{\text {w }}$ & $\begin{array}{c}\text { Broad } \\
\text { crop } \\
\text { labeling }\end{array}$ & $\begin{array}{c}\text { REI }^{v} \\
\text { (hours) }\end{array}$ & $\begin{array}{c}\text { Manufacturerl } \\
\text { Distributor }^{\mathrm{u}}\end{array}$ & Comments \\
\hline & oil, paraffinic & $\begin{array}{l}\text { Omni Oil, Omni } \\
\text { Supreme Spray }\end{array}$ & $6 \mathrm{E}(98 \%$ ai) & $\begin{array}{l}\text { Eriophyid, } \\
\text { Spider }\end{array}$ & $E, I, A$ & $\mathrm{O}$ & No & 4 & Helena & $\begin{array}{l}\text { Spray no more than } 4 \text { times } \\
\text { during the growing season; } \\
\text { use a two-week-minimum } \\
\text { application interval. }\end{array}$ \\
\hline & & Ultra-Fine ${ }^{(\mathbb{B})}$ Oil & (98.8\% ai) & Spider & $E, I, A$ & $\mathrm{G}, \mathrm{I}, \mathrm{O}$ & Yes & 4 & $\begin{array}{l}\text { Whitmire Micro- } \\
\text { Gen }\end{array}$ & \\
\hline & oil, petroleum & Saf-T-Side & EC (80.0\% ai) & $\begin{array}{l}\text { Eriophyid, } \\
\text { Spider }\end{array}$ & $E, I, A$ & $\mathrm{G}, \mathrm{O}$ & No & 4 & $\begin{array}{l}\text { Brandt } \\
\text { Consolidated }\end{array}$ & Total coverage important. \\
\hline & & $\begin{array}{l}\text { Omni Supreme } \\
\text { Spray (paraffin } \\
\text { base) }\end{array}$ & (98\% ai) & & $E, I, A$ & $\mathrm{O}$ & No & 4 & Helena & $\begin{array}{l}\text { For shade trees and } \\
\text { shrubs. }\end{array}$ \\
\hline & & $\begin{array}{l}\text { Damoil } \\
\text { PureSpray } \\
\text { Green, Ultra- } \\
\text { Pure }^{\mathrm{TM}} \text { Oil }\end{array}$ & (98\% ai) & & $E, I, A$ & $\mathrm{G}, \mathrm{O}, \mathrm{S}$ & Yes & 4 & $\begin{array}{l}\text { Drexel, Petro- } \\
\text { Canada, } \\
\text { Whitmire Micro- } \\
\text { Gen }\end{array}$ & $\begin{array}{l}\text { OMRI listed except } \\
\text { Damoil }^{\mathrm{TM}} \text {. }\end{array}$ \\
\hline & $\begin{array}{l}\text { oil, rosemary } \\
\text { and mint }\end{array}$ & EcoTrol $^{(B)}$ & EC (12\% ai) & $\begin{array}{l}\text { Eriophyid, } \\
\text { Spider, } \\
\text { Tarsonemid }\end{array}$ & & $\mathrm{G}, \mathrm{I}, \mathrm{O}, \mathrm{S}$ & Yes & 0 & EcoSMART & $\begin{array}{l}\text { "Minimum-risk" pesticide, } \\
\text { meets the requirements for } \\
\text { the USDA National Organic } \\
\text { Program. Good coverage } \\
\text { very important, leaves an } \\
\text { oily residue. }\end{array}$ \\
\hline & oil, soybean & $\begin{array}{l}\text { Golden Pest } \\
\text { Spray Oilim }\end{array}$ & $93 \%$ ai & Spider & $E, I, A$ & $\mathrm{G}, \mathrm{O}, \mathrm{S}$ & Yes & 4 & Stoller & OMRI listed. \\
\hline - soap & $\begin{array}{l}\text { soap } \\
\text { (potassium } \\
\text { salts of fatty } \\
\text { acids) }\end{array}$ & M-Pede ${ }^{(B)}$ & LC (49\% ai) & $\begin{array}{l}\text { Eriophyid, } \\
\text { Spider }\end{array}$ & $E, I, A$ & $\mathrm{G}, \mathrm{I}, \mathrm{O}$ & Yes & 12 & $\begin{array}{l}\text { Dow } \\
\text { AgroSciences }\end{array}$ & $\begin{array}{l}\text { OMRI listed. Thorough } \\
\text { coverage very important. } \\
\text { Do not use with sulfur or } \\
\text { within three days of a sulfur } \\
\text { application. }\end{array}$ \\
\hline
\end{tabular}


Table 1. Selected products for use in controlling mites on ornamental plants. (continued)

\begin{tabular}{|c|c|c|c|c|c|c|c|c|c|c|}
\hline $\begin{array}{l}\text { Mode } \\
\text { of } \\
\text { action } \\
\text { group }^{z}\end{array}$ & $\begin{array}{l}\text { Active } \\
\text { ingredient }\end{array}$ & Trade name(s) & Formulation(s) & $\begin{array}{c}\text { Mites } \\
\text { controlled }^{\mathrm{y}}\end{array}$ & $\begin{array}{c}\text { Mite } \\
\text { stages } \\
\text { controlled }^{\mathrm{x}} \\
\end{array}$ & Location $^{\text {w }}$ & $\begin{array}{c}\text { Broad } \\
\text { crop } \\
\text { labeling }\end{array}$ & $\begin{array}{c}\text { REI }^{v} \\
\text { (hours) }\end{array}$ & $\begin{array}{c}\text { Manufacturerl } \\
\text { Distributor }\end{array}$ & Comments \\
\hline sulfur & $\begin{array}{l}\text { sulfur, } \\
\text { micronized }\end{array}$ & Thiolux $^{(B)}$ Jet & $80 \mathrm{DF}$ & $\begin{array}{l}\text { Spider, } \\
\text { Tenuipalpid, }\end{array}$ & & G, On & No & 24 & Syngenta & $\begin{array}{l}\text { OMRI listed. Do not use } \\
\text { within two weeks of an oil } \\
\text { treatment. Complete } \\
\text { coverage important. }\end{array}$ \\
\hline
\end{tabular}

\footnotetext{
${ }^{\mathbf{z}}$ Mode of action classification scheme (version 6.1, Aug 2008 ) developed by the Insecticide Resistance Action Committee (IRAC) (http://www.irac-

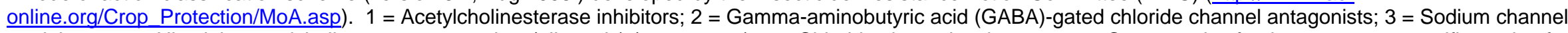

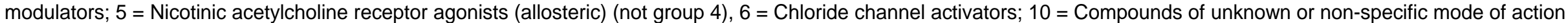

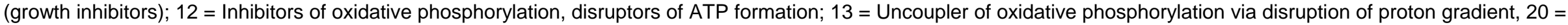

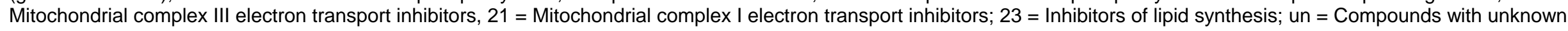
mode of action; - = not IRAC listed but may control mites physically (i.e., desiccation, membrane disruption, suffocation, etc.).

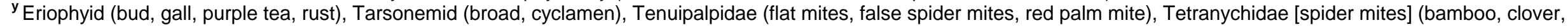
Lewis, southern red, spruce, tumid, twospotted).

${ }^{x} E=$ egg, $I=$ immatures, $A=$ adult

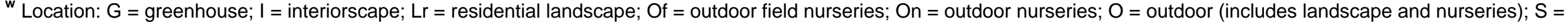
shadehouse.

${ }^{\mathrm{R}} \mathrm{REI}=$ restricted entry interval.

" See Table 2 for complete names and addresses.

$\mathrm{t}$ = restricted-use pesticide

s ai = active ingredient.

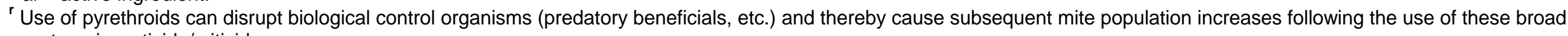
spectrum insecticide/miticides.

${ }^{\mathbf{q}}$ Can be used in IPM programs where predatory mites are used.

${ }^{\mathbf{p}}$ na $=$ not applicable.

${ }^{\circ} \mathrm{OMRI}=$ Organic Materials Review Institute (www.omri.org).
} 
Table 2. List of miticide manufacturers/distributors.

\begin{tabular}{|c|c|c|c|c|c|}
\hline Company/Division & Address & City & $\begin{array}{l}\text { Statel } \\
\text { Province }\end{array}$ & Zip Code & URL \\
\hline Bayer Environmental Science & 2 T.W. Alexander Drive & Research Triangle Park & NC & 27709 & http://www.bayerprocentral.com \\
\hline Brandt Consolidated Industries & 2935 S. Koke Mill Rd. & Springfield & IL & 62711 & http://www.brandtnews.com \\
\hline Cheminova & & Wayne & $\mathrm{NJ}$ & & http://www.cheminova.us.com \\
\hline Dow AgroSciences & 9330 Zionsville Rd & Indianapolis & IN & 46268 & http://www.dowagro.com \\
\hline Drexel Chemical & 1700 Channel Avenue & Memphis & $\mathrm{TN}$ & 38113 & http://www.drexchem.com \\
\hline EcoSMART/EcolPM & 318 Seaboard Lane, Ste 208 & Franklin & $\mathrm{TN}$ & 37067 & http://www.ecosmart.com \\
\hline Florikan E.S.A. & 1523 Edger Place & Sarasota & FL & 34240 & http://www.florikan.com \\
\hline $\begin{array}{l}\text { FMC/Agricultural Products } \\
\text { Group }\end{array}$ & 1735 Market Street & Philadelphia & PA & 19103 & http://www.fmcprosolutions.com \\
\hline Gowan & 370 Main Street & Yuma & $A Z$ & 85366 & http://www.gowanco.com \\
\hline Helena Chemical & 225 Schilling Blvd. & Collierville & $\mathrm{TN}$ & 38017 & http://www.helenachemical.com \\
\hline JH Biotech & 4951 Olivas Park Dr & Ventura & $\mathrm{CA}$ & 93003 & http://www.jhbiotech.com \\
\hline $\begin{array}{l}\text { Makhteshim-Agan of North } \\
\text { America (MANA) }\end{array}$ & $\begin{array}{l}4515 \text { Falls of Neuse Toad, Suite } \\
300\end{array}$ & Raleigh & NC & 27609 & http://www.manainc.com \\
\hline $\mathrm{OHP}$ & P.O. Box 230 & Mainland & PA & $19451-0230$ & http://ohp.com \\
\hline Petro-Canada & P.O. Box 2844 & Calgary & Alberta & T2P 3E3 & http://www.petro-canada.ca \\
\hline Prokoz & $\begin{array}{l}100 \text { North Point Center East, Suite } \\
330\end{array}$ & Alpharetta & GA & $30022-8242$ & http://www.prokoz.net/ \\
\hline Quali-Pro & $\begin{array}{l}4515 \text { Falls of Neuse Toad, Suite } \\
300\end{array}$ & Raleigh & NC & 27609 & http://www.Quali-pro.com \\
\hline Regal Chemical & 600 Branch Drive & Alpharetta & GA & 30004 & http://www.regalchem.com \\
\hline Scotts Company, The & 14111 Scottslawn Road & Marysville & $\mathrm{OH}$ & 43041 & http://www.scottsprohort.com \\
\hline SePRO & $\begin{array}{l}11550 \text { North Meridian Street, Suite } \\
600\end{array}$ & Carmel & IN & 46032 & http://www.sepro.com \\
\hline $\begin{array}{l}\text { Southern Agricultural } \\
\text { Insecticides }\end{array}$ & P.O. Box 218 & Palmetto & $\mathrm{FL}$ & 34221 & http://www.southernag.com \\
\hline Stoller Enterprises & $\begin{array}{l}4001 \text { W Sam Houston Pkwy N, } \\
\text { Suite } 100\end{array}$ & Houston & TX & 77043 & http://www.stollerusa.com \\
\hline
\end{tabular}


Table 2. List of miticide manufacturers/distributors.

\begin{tabular}{|c|c|c|c|c|c|}
\hline Company/Division & Address & City & $\begin{array}{l}\text { Statel } \\
\text { Province }\end{array}$ & Zip Code & URL \\
\hline Syngenta Professional Products & P.O. Box 18300 & Greensboro & NC & 27419 & http://www.syngentaprofessionalproducts.com \\
\hline United Phosphorus, Inc. & $\begin{array}{l}630 \text { Freedom Business Center, } \\
\text { Ste. } 402\end{array}$ & King of Prussia & $\mathrm{PA}$ & 19406 & http://www.upi-usa.com \\
\hline Valent Professional Products & 1701 Gateway Blvd., Suite 385 & Richardson & TX & 75080 & http://www.valentpro.com \\
\hline Wellmark International & $\begin{array}{l}1501 \text { E. Woodfield Rd., Suite } 200 \\
\text { West }\end{array}$ & Schaumburg & IL & 60173 & http://www.wellmarkinternational.com \\
\hline $\begin{array}{l}\text { Whitmire Micro-Gen Research } \\
\text { Laboratories }\end{array}$ & 3568 Tree Court Industrial Blvd. & St. Louis & $\mathrm{MO}$ & 63122 & http://www.wmmg.com \\
\hline
\end{tabular}

